\title{
SURGICAL CHALLENGES FACED BY TRAINEE SURGEONS- A PROSPECTIVE COMPARATIVE STUDY
}

\author{
Supriya Mushriff', Vandana Telgote², Kaynat Patel $^{3}$ \\ ${ }^{1}$ Assistant Professor, Department of Ophthalmology, Index Medical College Hospital and Research Centre. \\ 2 Professor, Department of Ophthalmology, Index Medical College Hospital and Research Centre. \\ ${ }^{3}$ Postgraduate Resident, Department of Ophthalmology, Index Medical College Hospital and Research Centre.
}

\begin{abstract}
BACKGROUND
ABSTRACT

Human resources like Doctors form the important part of all health systems. To achieve health objectives and goals, health resources should be distributed evenly among people irrespective of economic, social and geographical barriers.

The aim of this study is to evaluate the surgical challenges faced by trainee Ophthalmologists and to compare the efficacy of two different methods of training; step-by-step method and one-step method are the two ways of training practiced to train ophthalmologists. This study compares the two ways of training by evaluating complications at the end of surgeries performed by trainee surgeons.
\end{abstract}

Setting- Department of Ophthalmology, Index Medical College and Research Centre.

Study Design- A Prospective Comparative Study.

\section{MATERIALS AND METHODS}

Time Period- $1^{\text {st January }} 2017$ to $31^{\text {st }}$ March 2017, 3 months.

Cataract Surgery performed- Manual Small Incision Cataract Surgery performed within the above-mentioned period (80 SICS).

Study was divided into 2 Groups accordingly-

Group 1- Including residents that were trained with the 'step-by-step' method (44 SICS).

Group 2- Including residents that were trained with the 'one-step' method (36 SICS).

1. Residents were evaluated for the ease of performing surgical steps.

2. Complications were recorded in terms of premature entry, buttonholing, running away of capsulorhexis, rhexis tear, zonular dialysis/dehiscence, posterior capsular rent/rupture, iridodialysis, vitreous loss.

Both of the above-mentioned groups were compared according to various difficulties encountered in each approach and their management was done by the supervising surgeon. Patient was followed for 1 month. Data were listed and counted with Excel (Version 2007, Microsoft). Mean and Standard deviation was calculated. Chi-square and Student t-tests were used to assess statistical significance between groups.

\section{RESULTS}

44 surgeries were done in "step-by-step" group of which 18 patients were male and 26 were female; and 36 surgeries were done in "one-step" group of which 20 were male and 16 were female. Mean age group of patient in "step-by-step" group was $64.22 \pm 10.10$ years and $63.50 \pm 8.46$ years in "one-step" group. Overall complication rate when compared between 2 groups were more in "onestep" group, which was significant in contrast to when individual complication rates were compared between two groups.

\section{CONCLUSION}

Thus to conclude, learning surgery is always an uphill task for a trainee. A great degree of temperament, practice and skills are required before proficiency is achieved. In our study, 2 approaches were practiced in learning SICS- "step-by-step" and "one-step"; and "step-by-step" approach was found beneficial for the beginners.

\section{KEYWORDS}

SICS, Trainee Surgeon, Cataract, Post-Operative Complication.

HOW TO CITE THIS ARTICLE: Mushriff S, Telgote V, Patel K. Surgical challenges faced by trainee surgeons- a prospective comparative study. J. Evolution Med. Dent. Sci. 2017;6(50):3867-3871, DOI: 10.14260/Jemds/2017/835

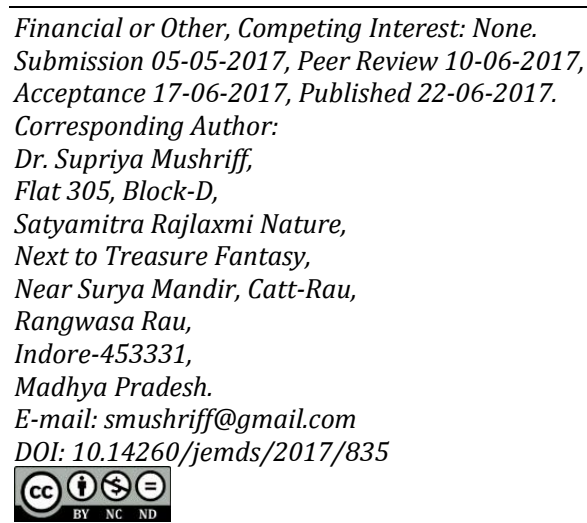

\begin{abstract}
BACKGROUND
Human resources like Doctors form the important part of all health systems.

To achieve health objectives and goals, health resources should be distributed evenly among people irrespective of economic, social and geographical barriers.

In Ophthalmology, age-related cataract is one of the important causes of all over the world. Growing population of developing countries and population ageing in both developing and developed countries will gradually increase the population with cataract-related blindness in near future. And to meet this requirement, more surgically trained Ophthalmologist are needed.
\end{abstract}


It is believed that there are more than 10,000 trained ophthalmologists in India.[1] India produces a large number of ophthalmologists annually. These trained professionals serve the needs of India and abroad. However, the quality of training infrastructure and facilities has never been systematically evaluated.

To accommodate this situation, effective and validated surgical training programs are required to ensure that residents in training achieve greater levels of competence in cataract surgery. Wet laboratory training and simulators play an increasingly important role as part of an organised surgical curriculum.[2,3] Surgical competency assessment tools are also being developed and integrated in ophthalmic training[4]; and in many residency programs, trainees' point of view of the quality of training they receive is investigated [57] as well as cataract surgery trainers' attitudes and techniques. ${ }^{[8]}$ India needs a large number of ophthalmologists to meet future demand. And a large number of training slots to increase surgically trained cataract surgeon.

The surgical portion of the training program is defined by a set of specifically outlined surgical numbers to allow for experience with the most common of ophthalmological procedures. Surgical skill of any surgeon depends upon Personality, Temperament, Surgical Aptitude, Trainer's Judgement and Structured Training Programme. Challenges faced by the beginners are Patient Selection, well versed with ECCE, Hand-Eye coordination and Step-by-Step approach.

Over the period, cataract surgery has evolved from couching to the latest femtosecond laser-assisted Microincision Cataract Surgery (MICS). Starting from couching it has travelled to Intracapsular Cataract Extraction (ICCE), Extracapsular Cataract Extraction, Small Incision Cataract Surgery (SICS), Phacoemulsification and Microincision Cataract Surgery.

Small Incision Cataract Surgery (SICS) is one of the cataract surgical techniques commonly used in developing countries as it is economical, have good visual outcome and is good for high volume cataract surgery.[1-3]

Small Incision Cataract Surgery (SICS) is often taught in stages, where a trainee performs a single part of the procedure several times in succession under the direct supervision of an experienced surgeon. When each step is mastered, the trainee then proceeds by learning the next step of the procedure. Other experienced tutors let residents try the whole procedure all at once. So far a number of teaching techniques have been described[9,10]; however, to the best of our knowledge there are no published studies in the literature directly comparing their efficacy on resident surgical outcomes.

Complications during Small Incision Cataract Surgery by trainee residents are Tunnel Incision/ Superficial/ Buttonholing, Tear at lateral edge, Premature entry, Running away of Capsulorhexis, Capsulorhexis Tear, Zonular Dialysis, Posterior Capsular rent, Iridodialysis and Vitreous Loss.

\section{Buttonholing}

- If the crescent knife can be seen very clearly, this indicates that the scleral layer is very thin $\rightarrow$ 'buttonhole.'

- A buttonhole can be corrected by making a deeper 'frown' incision and dissecting the tunnel in a deeper plane, starting at the opposite side of the buttonhole.

\section{Premature Entry}

- If the crescent knife is not visible during the incision, this indicates a 'premature entry.'

- Surgical complications such as iris trauma or iridodialysis, iris prolapse and not self-sealing tunnel.

- Manage a premature entry starting a shallow dissection at the other end of the tunnel. Suturing of the wound.

\section{Opening of Anterior Capsule}

- Can-opener technique.

- Capsulorhexis.

- Envelope.

Failing to complete the capsulorhexis, making a too-small CCC, and pulling residual anterior capsular tags can cause the posterior capsule to rupture.

\section{Nucleus Removal}

- Hydroexpression alone (Using an anterior chamber maintainer).

- Hydroexpression plus extraction (Using an irrigating vectis).

- Viscoexpression.

- $\quad$ Extraction alone (Using a 'fishhook' needle).

\section{Objective}

The aim of this study is to evaluate and compare the efficacy of two different training methods in resident-performed Small Incision Cataract Surgery (SICS) and assess their impact on complications in residents' performed surgery.

\section{MATERIALS AND METHODS}

Resident-performed cataract surgery by means of Small Incision Cataract Surgery (SICS) at a tertiary care centre from January 2017 to March 2017, were included in a prospective comparative study. The study was conducted in accordance with the tenets of the Declaration of Helsinki and was approved by the local institutional review board.

Analysis of all Small Incision Cataract Surgery (SICS) performed by ophthalmology residents from January 2017 to March 2017 in the Department of Ophthalmology, Index Medical College and Research Centre, was conducted. Data were collected by reviewing patient's electronic medical records. Data were listed and counted with Excel (Version 2007, Microsoft). Mean and Standard deviation was calculated. Chi-square and Student t-tests were used to assess statistical significance between groups.

Written informed consent to undergo cataract surgery was obtained from all patients and residents' participation in the operation was clearly disclosed. Operations performed by residents with previous surgical experience in other hospitals were excluded from this study. All surgical procedures were performed by 4 consecutive residents who did not have any previous experience as primary surgeon on intraocular surgeries.

\section{Inclusion Criterion}

Patients with cataract causing visual disturbance and no history of significant risk factors were eligible for resident training. 


\section{Exclusion Criterion}

Patients with ocular disease such as corneal opacity or irregularity, astigmatism greater than $2.5 \mathrm{D}$, severe dry eye syndrome, inadequate visualisation of the fundus, IOL power calculation less than 10.0 diopters (D) or more than $30.0 \mathrm{D}$, surgical complications on the fellow eye, and incomplete followup. Patients with a history of uveitis and current intraocular inflammation, uncontrollable glaucoma, proliferative diabetic retinopathy or retinal detachment.

Small Incision Cataract Surgery (SICS) was performed in all cases under Peribulbar block (Lignocaine 2\% + Bupivacaine $0.5 \%$ + Hyaluronidase) anaesthesia.

All procedures were supervised by one high-volume experienced surgeon, who had the priority to take over in case of intraoperative complications or where the resident surgeon was unable to complete the procedure.

Residents were categorised in two groups, Group A and Group B, according to the method of surgical training they received. The allocation of residents to the training methods was conducted randomly and was not affected by unknown factors such as preferences of their supervisors. All residents received equal traditional didactic and wet-lab training prior to operating on patient eyes. Group A residents were trained with a step-by-step method, performing a gradually increasing number of Small Incision Cataract Surgery (SICS) steps during the course of their training. Upon finishing each step successfully without any associated complications, the resident was allowed to proceed by learning the next step of the procedure.

Steps were taught in the same sequence as they appear in Small Incision Cataract Surgery (SICS) (sclera-corneal incisions; capsulorhexis; hydrodissection/hydrodelineation; nucleus prolapse and delivery; cortex removal by irrigation/aspiration; IOL insertion/removal of viscoelastic device). Group B residents received a one-step method training program, during which they proceeded to perform the entire operation in one step.

Outcomes of surgeries performed by Group A residents were compared with those of cases performed by their Group B counterparts. Primary outcomes were the incidence of main complications defined by premature entry, buttonholing, running away of capsulorhexis, rhexis tear, zonular dialysis/dehiscence, posterior capsular rent/rupture, iridodialysis and vitreous loss.

\section{Steps Performed during Surgery}

- $\quad$ All cases included in the study was done by manual SICS by the Residents.

- A 6 mm Sclerocorneal tunnel was made.

- Capsulorhexis was done in all cases.

- Release/relaxing incisions were made over capsulorhexis margin as and when required.

- Nuclei was prolapsed in Anterior Chamber (AC).

- Methylcellulose was injected in Anterior Chamber (AC).

- Nuclei was delivered by Visco-expression method.

- IOL was implanted in the bag.

- Residents were allocated into two groups according to the method that was applied during training in cataract surgery.
- Group A- Including residents that were trained with "step-by-step" method (44 SICS).

- Group B- Including residents that were trained with "one-step" method (36 SICS).

- The SICS procedures in both groups were supervised by one attending surgeon.

- Supervisors had the priority to stop a resident's attempt in situations such as anterior capsular runaway, inability to complete Continuous Curvilinear Capsulorhexis (CCC), failure to separate the nucleus after three trials, any dangerous movement in the eye, difficulty keeping a stable anterior chamber depth during any step, and the occurrence of intraoperative complications.

- Residents were evaluated for the ease of performing surgical steps.

- Complications were recorded in terms of premature entry, buttonholing, running away of capsulorhexis, rhexis tear, zonular dialysis/dehiscence, posterior capsular rent/rupture, iridodialysis and vitreous loss.

- Both of the above-mentioned groups were compared according to various difficulties encountered in each approach and is managed accordingly.

\section{RESULTS}

- Examination- On 1st post-operative day, 15th day, 1 month.

- Post-operative recovery- Uneventful.

- Findings- Minimum conjunctival reaction- 10 patients.

- Intraocular pressure- Within 10 - $20 \mathrm{~mm}$ mercury. AC reaction- no cells and flare- from 15 th day. Best corrected vision- 6/9-6/12.

- Group A- (STEP-BY-STEP).

- Group B-(ONE-STEP).

\begin{tabular}{|c|c|c|c|}
\hline & Group A & Group B & P value \\
\hline $\begin{array}{c}\text { Small Incision Cataract } \\
\text { Surgery }\end{array}$ & 44 & 36 & \\
\hline Gender & $18 \mathrm{M} / 26 \mathrm{~F}$ & $20 \mathrm{M} / 16 \mathrm{~F}$ & 0.19 \\
\hline Mean Age (in years) & $64.22 \pm 10.10$ & $63.5 \pm 8.46$ & 0.34 \\
\hline \multicolumn{2}{|r|}{ Table 1. Demographic Data } \\
\hline
\end{tabular}

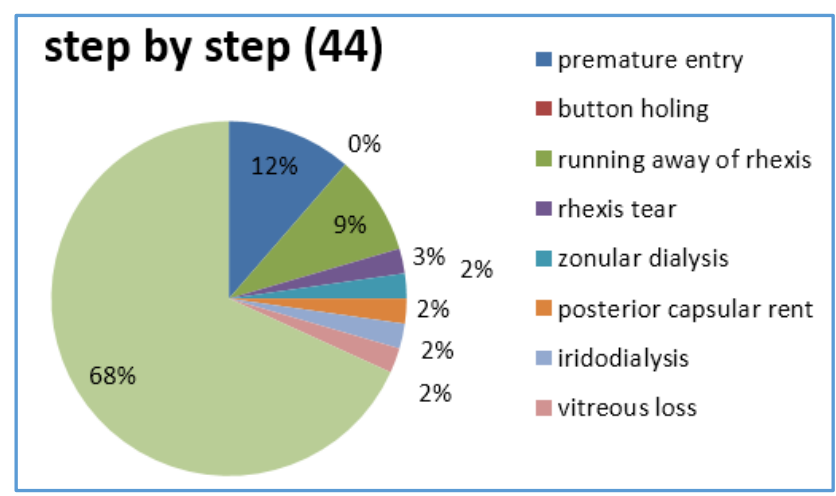

Graph 1. Distribution of Complications in Group A 


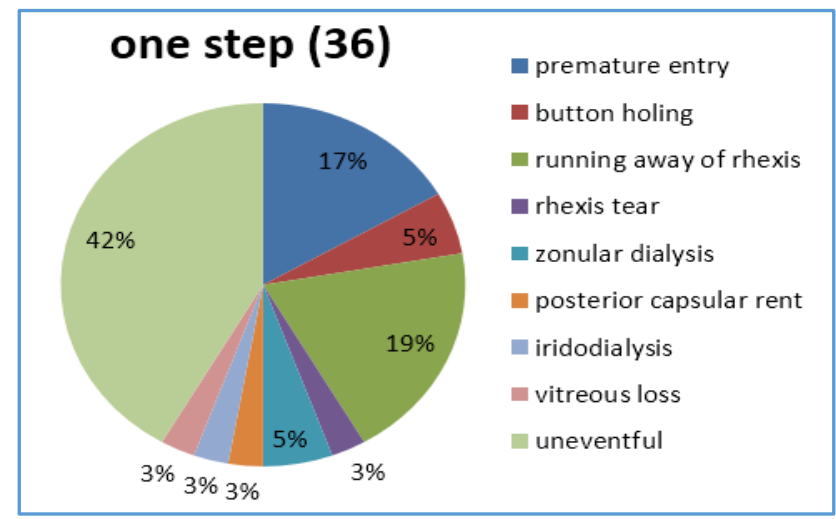

Graph 2. Distribution of Complications in Group B

\begin{tabular}{|c|c|c|c|c|c|}
\hline & $\begin{array}{c}\text { Step by } \\
\text { Step } \\
\text { (44) }\end{array}$ & $\begin{array}{c}\text { One } \\
\text { Step } \\
\text { (36) }\end{array}$ & $\begin{array}{c}\text { Step } \\
\text { by } \\
\text { Step \% }\end{array}$ & $\begin{array}{c}\text { One } \\
\text { Step } \\
\text { \% }\end{array}$ & P value \\
\hline $\begin{array}{c}\text { Premature } \\
\text { entry }\end{array}$ & 5 & 6 & 11.36 & 16.67 & 0.493209 \\
\hline $\begin{array}{c}\text { Button- } \\
\text { holing }\end{array}$ & 0 & 2 & 0 & 5.55 & 0.113333 \\
\hline $\begin{array}{c}\text { Running } \\
\text { away of } \\
\text { rhexis }\end{array}$ & 4 & 7 & 9.09 & 19.44 & 0.180963 \\
\hline Rhexis tear & 1 & 1 & 2.27 & 2.78 & 0.885544 \\
\hline $\begin{array}{c}\text { Zonular } \\
\text { dialysis }\end{array}$ & 1 & 2 & 2.27 & 5.56 & 0.265959 \\
\hline $\begin{array}{c}\text { Posterior } \\
\text { capsular } \\
\text { rent }\end{array}$ & 1 & 1 & 2.27 & 2.78 & 0.885544 \\
\hline Iridodialysis & 1 & 1 & 2.27 & 2.78 & 0.885544 \\
\hline $\begin{array}{c}\text { Vitreous } \\
\text { loss }\end{array}$ & 1 & 1 & 2.27 & 2.78 & 0.885544 \\
\hline Uneventful & 30 & 15 & 68.18 & 41.67 & 0.01739 \\
\hline $\begin{array}{c}\text { Table 2. Comparison of Rate of Various } \\
\text { Complications in Group A and Group B }\end{array}$ \\
\hline
\end{tabular}

- Complication rate of the two groups was compared by Chi-Square test.

- Group A- (Step-by-Step).

- 14 cases were complicated/eventful out of 44 cases performed.

- Incidence or rate of complications in Group $A=31.81 \%$.

- Group B- (One-Step).

- 21 cases were complicated/eventful out of 36 cases performed.

- Incidence or rate of complications in Group B=58.33\%.

- Premature entry, running away of capsulorhexis, buttonholing, zonular dialysis as we can see in above table are more in one-step group when compared to step-by-step group, but were insignificant, $[\mathrm{P}$ value $>$ 0.05].

- A statistically significant difference $(P=0.01739)$ was noted in the overall complications rate between the two groups yielding an incidence of $31.81 \%$ in Step-by-Step Group and 58.33\% in One-Step Group respectively, ( $<$ 0.05).

- When individual complication rate was compared between the two groups, they were not shown to differ statistically significant with $(\mathrm{P}>0.05)$.

\section{DISCUSSION}

- Till now no study is seen to compare our results comparing the efficacy of both methods in Small Incision Cataract Surgery. But we have found different studies comparing efficacy of step-by-step method and one-step method in Phacoemulsification. To evaluate and compare the efficacy of two different training methods in resident-performed phacoemulsification surgery, A. Tzamalis et al at Greece, performed 502 eyes of 467 patients. A statistically significant difference $(p=0.0032)$ was noted in the main complications rate between the two groups, yielding a mean of $17.3 \%$ in Step-by-Step Group and 7.25\% in One-Step Group respectively.

- Other intraoperative complications, such as anterior capsule tear or extended capsulorhexis were not shown to differ statistically significantly between study groups $(\mathrm{p}>0.05)$.

- There are large number of studies, in which the incidence of intraoperative complications in resident performing phacoemulsification surgeries was assessed. Hashemi et al demonstrated that among 500 cases operated by residents, $51(10.2 \%)$ developed vitreous loss and $48(9.6 \%)$ developed posterior capsule rupture and vitreous loss.[11] They concluded that direct attending supervision and careful case selection for the level of cataract surgery residency were of utmost importance in avoiding sight-threatening complications. Pokroy et al, trying to determine whether virtual surgery simulator training improves actual resident cataract surgery performance, found that capsular tear rates for the non-simulator and simulator groups were $8.8 \%$ and $10 \%$, respectively for the first 25 cases.[12] Carricondo et al, studying resident-performed phacoemulsification surgery indicated a statistically significant difference in PCR rates between the first 40 cases per resident and the total number of operations yielding a mean of $9.65 \%$ in the first 40 operations, data comparable to our results.[13] Several other studies showed lower complication rates in residents training programs varying from $3 \%$ to $7 \% .{ }^{[9-16]}$

- This difference may be attributed to the fact that residents included in these studies were not in all cases surgically naive and the complications rate was calculated for a larger amount of phacoemulsification cases performed by each resident, which could possibly lower the cumulative complications rate according to the learning curve.

In the modern era with increase in advancement in health sector population is increasing. India is the $2^{\text {nd }}$ most populous country after China with a large young population. Within few decades as this young population will age, age-related Cataract will be the most common cause of visual impairment among them. Cataract is one of the most common day procedure performed worldwide, raising its number to 15 million annually. To fulfil this, a large number of trained ophthalmologists with surgical skills are needed worldwide to attain the goal of Vision 20-20. Nearly 900 ophthalmologists are trained in India every year. It has been estimated that India needs 25,000 ophthalmologists by 2020 to achieve the goals of Vision 20-20: The Right to Sight.[17]

This would entail an additional 300 training slots every year to meet the projected human resource needs.[17] 
These training slots should improvise their training methodology, as Ophthalmology is increasing in its innovations and complexity. Though Phacoemulsification is the most common type of Cataract surgery nowadays, still Small Incision Cataract surgery practiced in developing country like India due to resources constrained, high volume of patients and as a part of training programme.

There is a high mismatch ratio among training institutes responsible for training budding ophthalmologists in surgical skills, in giving appropriate number of cases, teaching facilities like videos, CD's and wet labs.

The future of training should look at combining conventionally taught components with the use of up-to-date multimedia resources including books, course guides, videotapes, audiotapes, television, e-conferencing and discussion groups as well as access to web-based learning.[18]

Small Incision Cataract Surgery should be taught step by step under direct supervision of a trained surgeon. Therefore, in our study, we attempted to assess and compare the efficacy of the above-mentioned different training methods in resident-performed Small Incision Cataract Surgery. Certainly, there are some limitations of our study. First is small sample size, i.e. 80 cases. Further study is required for comparison b/w two groups with large sample size.

We have not taken surgery time into consideration, as this study was of beginners. Taking time constraints can induce anxiety among beginners and also efficacy of any training procedure is calculated by the complications occurring during surgery.

Our study was retrospective in design and there was single attending surgeon for review and supervision. In addition, confounding data such as ocular comorbidities, cataract characteristics of patients and other possible risk factors were not accounted for in this study.

\section{CONCLUSION}

In conclusion, our study signifies the importance of step-bystep approach for surgical training of the budding ophthalmologists and improving their surgical competencies. By doing this, we can decrease surgical complication rates. It also concludes that newer approaches should be applied for training of surgeons in addition to conventional techniques, thus lowering their learning curve. In the end, learning surgery is always an uphill task for a trainee. A great degree of temperament, practice and skills are required before proficiency is achieved. A "Step-by-Step" approach is beneficial for the beginners.

\section{REFERENCES}

[1] Kumar R. Ophthalmic manpower in India - need for a serious review. Int Ophthalmol 1993;17(5):269-75.

[2] Henderson BA, Grimes KJ, Fintelmann RE, et al. Stepwise approach to establishing an ophthalmology wet laboratory. Journal of Cataract and Refractive Surgery 2009;35(6):1121-8.

[3] McCannel CA, Reed DC, Goldman DR. Ophthalmic surgery simulator training improves resident performance of capsulorhexis in the operating room. Ophthalmology 2013;120(12):2456-61.

[4] Golnik C, Beaver H, Gauba V, et al. Development of a new valid, reliable, and internationally applicable assessment tool of residents competence in ophthalmic surgery. Transactions of the American Ophthalmological Society 2013;111:24-33.

[5] Mostafaei A, Hajebrahimi S. Perceived satisfaction of ophthalmology residents with the current Iranian ophthalmology curriculum. Clinical Ophthalmology 2011;5:1207-10.

[6] Gogate P, Deshpande M, Dharmadhikari S. Which is the best method to learn ophthalmology? Resident doctors perspective of ophthalmology training. Indian Journal of Ophthalmology 2008;56(5):409-12.

[7] McDonnell PJ, Kirwan TJ, Brinton GS, et al. Perceptions of recent ophthalmology residency graduates regarding preparation for practice. Ophthalmology 2007;114(2):387-91.

[8] Alexander P, Matheson D, Baxter J, et al. United Kingdom national cataract training survey. Journal of Cataract and Refractive Surgery 2012;38(3):533-8.

[9] Briszi A, Prahs P, Hillenkamp J, et al. Complication rate and risk factors for intraoperative complications in resident-performed phacoemulsification surgery. Graefe's Archive for Clinical and Experimental Ophthalmology 2012;250(9):1315-20.

[10] Ophthalmology/ Blindness Control Section, Government of India. National programme for control of blindness- India. Vision 2020: the right to sight. Plan of action, ophthalmology/ blindness control section directorate general of health services, ministry of health \& family welfare, government of India, New Delhi, India 2002:1-32.

[11] Hashemi H, Mohammadpour M, Jabbarvand H, et al. Incidence of and risk factors for vitreous loss in resident-performed phacoemulsification surgery. Journal of Cataract and Refractive Surgery 2013;39(9):1377-82.

[12] Pokroy R, Du E, Alzaga A, et al. Impact of simulator training on resident cataract surgery. Graefe's Archive for Clinical and Experimental Ophthalmology 2013;251(3):777-81.

[13] Carricondo PC, Fortes ACFM, Mourao PDC, et al. Senior resident phacoemulsification learning curve. Arquivos Brasileiros de Oftalmologia 2010;73(1):66-9.

[14] Blomquist PH, Morales ME, Tong L, et al. Risk factors for vitreous complications in resident-performed phacoemulsification surgery. Journal of Cataract and Refractive Surgery 2012;38(2):208-14.

[15] Belyea DA, Brown SE, Rajjoub LZ. Influence of surgery simulator training on ophthalmology resident phacoemulsification performance. Journal of Cataract and Refractive Surgery 2011;37(10):1756-61.

[16] Blomquist PH, Sargent JW, Winslow HH. Validation of najjar-awwad cataract surgery risk score for resident phacoemulsification surgery. Journal of Cataract \& Refractive Surgery 2010;36(10):1753-7.

[17] Rutar T, Porco TC, Naseri A. Risk factors for intraoperative complications in resident performed phacoemulsification surgery. Ophthalmology 2009; 116(3):431-6.

[18] McKimm J, Jollie C, Cantillon P. ABC of learning and teaching: web based learning. BMJ 2003; 326(7394): 870-3. 